Numerical-analytic method for investigating boundary value problems for impulsive differential equations

\author{
I. I. Korol
}




\title{
NUMERICAL-ANALYTIC METHOD FOR INVESTIGATING BOUNDARY VALUE PROBLEMS FOR IMPULSIVE DIFFERENTIAL EQUATIONS
}

\author{
I. I. KOROL
}

Received 25 March, 2008

\begin{abstract}
The new numerical-analytic method for investigation of the nonlinear periodical impulsive systems of differential equations is substantiated. The problems of existing and approximate construction of the solutions are studied and the error estimates are obtained.
\end{abstract}

2000 Mathematics Subject Classification: 34A37, 34B05

Keywords: numerical-analytic method, boundary value problem, impulsive differential equation

\section{INTRODUCTION}

There are numerous methods for investigating and approximate constructing of the solutions of the boundary value problems for ordinary differential equations and, in particular, for differential equations with impulses (see, e.g., $[1,2,5,6]$ and the references therein). From the large variety of them, we can distinguish the numericalanalytic method of successive approximations developed in $[5,6]$ where the problem on the existence and approximate construction of periodic solutions of the impulsive differential system of the form

$$
x^{\prime}(t)=f(t, x), \quad t \neq \tau_{i}, \quad x\left(\tau_{i}+0\right)-x\left(\tau_{i}\right)=I_{i}(x) .
$$

is studied on the assumption that $f$ is a the time-periodic function. Another important assumption guaranteeing the applicability of the approach of the works mentioned requires that $f$ and $I_{i}$ should satisfy the Lipschitz conditions with sufficiently small constants.

In this paper we attempt to generalise the numerical-analytic method of successive approximations [5,6] to nonlinear systems of differential equations with impulse action of the form

$$
x^{\prime}(t)=A(t) x+f(t, x), \quad t \neq \tau_{i}, \quad x\left(\tau_{i}+0\right)-x\left(\tau_{i}\right)=B_{i} x+I_{i}(x),
$$

in the cases where there exist a nontrivial solutions of the corresponding linear boundary value problem. The Lipschitz conditions concerns not all right-hand side term but only the non-linearity. 


\section{REGULARISATION OF LINEAR IMPULSIVE BOUNDARY VALUE PROBLEMS}

Let us first consider a linear inhomogeneous system of differential equations with impulse action

$$
\begin{gathered}
x^{\prime}(t)=A(t) x+h(t), \quad t \in[a, b] \backslash\left\{\tau_{1}, \tau_{2}, \ldots, \tau_{p}\right\}, \\
x\left(\tau_{i}+0\right)-x\left(\tau_{i}\right)=B_{i} x\left(\tau_{i}\right)+a_{i}, \quad i=1, \ldots, p,
\end{gathered}
$$

under the linear functional boundary conditions

$$
l x=\alpha,
$$

where $A(\cdot)$ and $h(\cdot)$ are, respectively, $(n \times n)$-matrix and $(n \times 1)$-vector functions that arepiecewise continuous, with discontinuity of the first kind at $t=\tau_{i}, i=1,2, \ldots, p$. The second relation in (2.1) determines the jumps of the solutions. Here $B_{i}, i=$ $1,2, \ldots, p$, are $(n \times n)$-constant matrices such that

$$
\operatorname{det}\left(\mathbb{1}_{n}+B_{i}\right) \neq 0, \quad i=1,2, \ldots, p,
$$

$\left\{a_{1}, a_{2}, \ldots, a_{p}, \alpha\right\} \subset \mathbb{R}^{n}$ are constant column vectors, and $a \leq \tau_{1}<\tau_{2}<\cdots<\tau_{p} \leq$ $b$. Equality (2.2), where $l$ is an $n$-dimensional bounded linear vector functional, determines the boundary conditions.

Let $P C\left([a, b] \backslash\left\{\tau_{i}\right\}_{i=1}^{p}, \mathbb{R}^{n}\right)$ stand for the space of functions $x:[a, b] \rightarrow \mathbb{R}^{n}$ which are continuous from the left with discontinuity of the first kind at the points $\tau_{i}$, $i=1, \ldots, p$ endowed with the norm $\|x\|=\sup \{|x(t)|: t \in[a, b]\}$. Moreover, let $P C_{\text {loc }}^{1}\left([a, b] \backslash\left\{\tau_{i}\right\}_{i=1}^{p}, \mathbb{R}^{n}\right)$ denote the set of functions $x \in P C\left([a, b] \backslash\left\{\tau_{i}\right\}_{i=1}^{p}, \mathbb{R}^{n}\right)$ which are continuously differentiable on every open interval contained in $[a, b] \backslash$ $\left\{\tau_{i}\right\}_{i=1}^{p}$. A function $x \in P C_{\mathrm{loc}}^{1}\left([a, b] \backslash\left\{\tau_{i}\right\}_{i=1}^{p}, \mathbb{R}^{n}\right)$ is said to be a solution of the problem (2.1), (2.2) if it satisfies the equalities (2.1) and verifies also the boundary condition (2.2).

According to the Riesz theorem [3], for an arbitrary linear bounded vector functional $l: P C\left([a, b] \backslash\left\{\tau_{i}\right\}_{i=1}^{p}, \mathbb{R}^{n}\right) \rightarrow \mathbb{R}^{n}$, there exist a matrix function of bounded variation $C(\cdot)$ such that by means of Riemann-Stieltjes integral we can represent the boundary condition (2.2) in the form

$$
\int_{a}^{b}[d C(t)] x(t)=\alpha
$$

It is known [5] that solution $x\left(t, x_{0}\right)$ of impulsive differential system (2.1) having the initial value $x\left(a, x_{0}\right)=x_{0}$ is of the form

$$
x\left(t, x_{0}\right)=X(t) x_{0}+\int_{a}^{t} X(t, s) h(s) d s+\sum_{a \leq \tau_{i}<t} X\left(t, \tau_{i}+0\right) a_{i},
$$

where $X(\cdot)$ is a fundamental matrix for the linear homogeneous impulsive system

$$
x^{\prime}(t)=A(t) x, \quad t \notin\left\{\tau_{i}\right\}_{i=1}^{p}, \quad x\left(\tau_{i}+0\right)-x\left(\tau_{i}\right)=B_{i} x, \quad i=1,2, \ldots, p .
$$


Recall that the matrix function $X$ has the properties* $X(a)=\mathbb{1}_{n}, X(t)=X(t, a)$, where $X(t, s)=X(t) X^{-1}(s)$, and $X\left(t, \tau_{i}+0\right)=X\left(t, \tau_{i}\right)\left(\mathbb{1}_{n}+B_{i}\right)^{-1}, i=1,2, \ldots, p$.

While substituting (2.3) into the boundary conditions (2.2) we can see that the initial value $x_{0}$ of the solution $x\left(t, x_{0}\right)$ of the boundary value problem (2.1), (2.2) must satisfy the equation

$$
G x_{0}=\alpha-\int_{a}^{b} Z(s) h(s) d s-\sum_{i=1}^{p} Z\left(\tau_{i}+0\right) a_{i} .
$$

Here $Z(s)=\int_{s}^{b}[d C(t)] X(t, s)$ and $G=Z(a)=l X=\int_{a}^{b}[d C(t)] X(t)$.

In a noncritical case [1] (when a linear homogeneous impulsive system (2.4) does not have a nontrivial solutions), the algebraic system (2.5) has a unique solution

$$
x_{0}=G^{-1}\left(\alpha-\int_{a}^{b} Z(s) h(s) d s-\sum_{i=1}^{p} Z\left(\tau_{i}+0\right) a_{i}\right),
$$

which is the initial value of a unique solution of the problem (2.1), (2.2)

$$
\begin{aligned}
x(t)=X(t) G^{-1} \alpha+\int_{a}^{t} X(t, s) h(s) d s-X(t) G^{-1} \int_{a}^{b} Z(s) h(s) d s & \\
& \quad+\sum_{a \leq \tau_{i}<t} X\left(t, \tau_{i}+0\right) a_{i}-X(t) G^{-1} \sum_{i=1}^{p} Z\left(\tau_{i}+0\right) a_{i} .
\end{aligned}
$$

Further we will consider a critical case [1], i. e., the case where

(A) There exist $k, 1 \leq k \leq n$, linearly independent solutions of the linear homogeneous impulsive boundary value problem

$$
\begin{gathered}
x^{\prime}(t)=A(t) x, \quad t \notin\left\{\tau_{i}\right\}_{i=1}^{p}, \\
x\left(\tau_{i}+0\right)-x\left(\tau_{i}\right)=B_{i} x\left(\tau_{i}\right), \quad i=1,2, \ldots, p ; \\
l x=0 .
\end{gathered}
$$

Lemma 1. Assume that there exist $k$ linearly independent solutions of the linear homogeneous boundary value problem with impulse action (2.6). Then for an arbitrary function $h(t)$ there exists a function $H(t)$ such that inhomogeneous impulsive differential system

$$
\begin{gathered}
x^{\prime}(t)=A(t) x+h(t)+H(t), \quad t \notin\left\{\tau_{1}, \tau_{2}, \ldots, \tau_{p}\right\}, \\
x\left(\tau_{i}+0\right)-x\left(\tau_{i}\right)=B_{i} x+a_{i}, \quad i=1,2, \ldots, p,
\end{gathered}
$$

possesses a $k$-parametric family of solutions which satisfy the boundary condition (2.2).

${ }^{*}$ Here and below, $\mathbb{1}_{n}$ stands for the unit matrix of dimension $n$. 
Proof. It is known [1] that the boundary value problem (2.7), (2.2) has a solution if and only if the condition

$$
P_{G^{*}}\left(\alpha-\int_{a}^{b} Z(s) h(s) d s-\int_{a}^{b} Z(s) H(s) d s-\sum_{i=1}^{p} Z\left(\tau_{i}+0\right) a_{i}\right)=0
$$

is fulfilled, ${ }^{\dagger}$ where $P_{G^{*}}: \mathbb{R}^{n} \rightarrow \operatorname{ker} G^{*}$ (resp., $P_{G}: \mathbb{R}^{n} \rightarrow \operatorname{ker} G$ ) is an orthoprojector to the null space $\operatorname{ker} G^{*}=\left\{z: z \in \mathbb{R}^{n}, z G^{*}=0\right\}\left(\right.$ resp., $\left.\operatorname{ker} G=\left\{y: y \in \mathbb{R}^{n}, G y=0\right\}\right)$ of $G^{*}$ (resp., $G$ ).

We denote by the symbol $P_{G ; k}$ the $(n \times k)$-matrix whose columns are the linearly independent columns of $P_{G}$ constituting a basis in $\operatorname{ker} G$. Similarly, by $P_{G^{*} ; k}$ we denote the $(k \times n)$-matrix whose rows are the linearly independent rows of the matrix $P_{G^{*}}$ constituting a basis in $\operatorname{ker} G^{*}$. Thus,

$$
\operatorname{rank} P_{G}=\operatorname{rank} P_{G ; k}=\operatorname{rank} P_{G^{*}}=\operatorname{rank} P_{G^{*} ; k}=k .
$$

Let us set

$$
H(t):=Z^{*}(t)\left(P_{G_{k}^{*}}\right)^{*} R_{1}^{-1} P_{G_{k}^{*}}\left(\alpha-\int_{a}^{b} Z(s) h(s) d s-\sum_{a \leq \tau_{i}<b} Z\left(\tau_{i}+0\right) a_{i}\right)
$$

for all $t \in[a, b]$, where $R_{1}:=P_{G^{*} ; k} R_{2}\left(P_{G_{k}^{*}}\right)^{*}$ and

$$
R_{2}:=\int_{a}^{b} Z(\tau) Z^{*}(\tau) d \tau
$$

One can see that condition (2.8) is fulfilled if $H(t)$ is of the form (2.9). The solution of the impulsive differential system (2.7) is

$$
\begin{aligned}
& x(t)=X(t) x_{0}+\int_{a}^{t} X(t, s) h(s) d s+\sum_{a \leq \tau_{i}<t} X\left(t, \tau_{i}+0\right) a_{i} \\
& +\int_{a}^{t} X(t, s) Z^{*}(s) d s\left(P_{G_{k}^{*}}\right)^{*} R_{1}^{-1} P_{G^{*} ; k}\left(\alpha-\int_{a}^{b} Z(s) h(s) d s-\sum_{i=1}^{p} Z\left(\tau_{i}+0\right) a_{i}\right) .
\end{aligned}
$$

Substituting (2.10) into (2.2) we can see that $x(t)$ satisfy the boundary condition if and only if the initial value $x_{0}$ is a solution of the algebraic system

$$
G x_{0}=\left(\mathbb{1}_{n}-R_{2} P_{G_{k}^{*}}^{*} R_{1}^{-1} P_{G_{k}^{*}}\right)\left(\alpha-\int_{a}^{b} Z(s) h(s) d s-\sum_{i=1}^{p} Z\left(\tau_{i}+0\right) a_{i}\right) .
$$

Since $P_{G ; k}\left(\mathbb{1}_{n}-R_{2} P_{G_{k}^{*}}^{*} R_{1}^{-1} P_{G^{*} ; k}\right)=0$, the system (2.11) is solvable and its general solution is of the form $[1,7]$

\footnotetext{
${ }^{\dagger}$ Here and below, we identify projectors with the corresponding matrices.
} 


$$
\begin{aligned}
& x_{0}=P_{G ; k} \xi \\
+ & G^{+}\left(\mathbb{1}_{n}-R_{2} P_{G_{k}^{*}}^{*} R_{1}^{-1} P_{G^{*} ; k}\right)\left(\alpha-\int_{a}^{b} Z(s) h(s) d s-\sum_{i=1}^{p} Z\left(\tau_{i}+0\right) a_{i}\right),
\end{aligned}
$$

where $\xi$ is an arbitrary $k$-dimensional column, $G^{+}$is a unique Moore-Penrose generalised inverse $(n \times n)$-matrix [4,7]. If substitute the initial value $x_{0}$ given by (2.12) in representation (2.10) we obtain the general solution of the boundary value problem boundary value problem (2.2), (2.7):

$$
\begin{aligned}
x(t) & =X(t) P_{G ; k} \xi \\
& +X(t) G^{+}\left(\mathbb{1}_{n}-R_{2} P_{G_{k}^{*}}^{*} R_{1}^{-1} P_{G_{k}^{*}}\right)\left(\alpha-\int_{a}^{b} Z(\tau) h(\tau) d \tau-\sum_{i=1}^{p} Z\left(\tau_{i}+0\right) a_{i}\right) \\
& +\int_{a}^{t} X(t, s) h(s) d s+\sum_{a \leq \tau_{i}<t} X\left(t, \tau_{i}+0\right) a_{i} \\
& +\int_{a}^{t} X(t, s) Z^{*}(s) d s P_{G_{k}^{*}}^{*} R_{1}^{-1} P_{G^{*} ; k}\left(\alpha-\int_{a}^{b} Z(\tau) h(\tau) d \tau-\sum_{i=1}^{p} Z\left(\tau_{i}+0\right) a_{i}\right) .
\end{aligned}
$$

Finally we can rewrite it in the form

$$
\begin{aligned}
x(t, \xi)=X(t) P_{G ; k} \xi+X(t)\left(G^{+}\right. & \left.+\left(R(t)-G^{+} R_{2}\right) P_{G_{k}^{*}}^{*} R_{1}^{-1} P_{G^{*} ; k}\right) \alpha \\
& +\int_{a}^{b} L(t, s) h(s) d s+\sum_{i=1}^{p} L\left(t, \tau_{i}+0\right) a_{i},
\end{aligned}
$$

where

$$
R(t)=\int_{a}^{t} X(s)^{-1} Z^{*}(s) d s
$$

and

$$
L(t, s)= \begin{cases}X(t, s)-X(t)\left(G^{+}+\left(R(t)-G^{+} R_{2}\right) P_{G_{k}^{*}}^{*} R_{1}^{-1} P_{G_{k}^{*}}\right) Z(s), & 0 \leq s \leq t \leq b, \\ -X(t)\left(G^{+}+\left(R(t)-G^{+} R_{2}\right) P_{G_{k}^{*}}^{*} R_{1}^{-1} P_{G_{k}^{*}}\right) Z(s), & 0 \leq t<s \leq b .\end{cases}
$$

Considering (2.13), we conclude that the lemma is proved.

\section{THE BOUNDARY VALUE PROBLEM FOR NONLINEAR IMPULSIVE DIFFERENTIAL EQUATIONS IN A CRITICAL CASE}

Now we consider the nonlinear system of differential equations with impulse action

$$
\begin{gathered}
x^{\prime}(t)=A(t) x+f(t, x), \quad t \notin\left\{\tau_{i}\right\}_{i=1}^{p}, \\
x\left(\tau_{i}+0\right)-x\left(\tau_{i}\right)=B_{i} x\left(\tau_{i}\right)+I_{i}\left(x\left(\tau_{i}\right)\right),
\end{gathered}
$$


under the boundary condition (2.2). We will investigate a critical case, i. e., the case, when the condition (A) is fulfilled.

We suppose that, for $t \in[a, b], x \in D \subset \mathbb{R}^{n}$, where $D$ is a closed and bounded domain, the following conditions hold:

(B) $A(t), f(t, x)$ are continuous or piecewise continuous for $t$ with discontinuity of the first kind for $t=\tau_{i}$ respectively $(n \times n)$-matrix function and $(n \times 1)$ vector function, $B_{i}$ are $(n \times n)$-constant matrices such that $\operatorname{det}\left(\mathbb{1}_{n}+B_{i}\right) \neq 0$, $a_{i}, \alpha$ are the $n$-dimensional column vectors of constants, $\tau_{0}<a \leq \tau_{1}<\tau_{2}<$ $\cdots<\tau_{p} \leq b, l$ is $n$-dimensional bounded linear vector functional, and the following inequalities hold:

$$
\begin{gathered}
|f(t, x)| \leq M(t), \quad\left|I_{i}(x)\right| \leq m_{i}, \\
\left|f\left(t, x^{\prime}\right)-f\left(t, x^{\prime \prime}\right)\right| \leq K(t)\left|x^{\prime}-x^{\prime \prime}\right|, \\
\left|I_{i}\left(x^{\prime}\right)-I_{i}\left(x^{\prime \prime}\right)\right| \leq K_{i}\left|x^{\prime}-x^{\prime \prime}\right|,
\end{gathered}
$$

where $m_{i}$ and $K_{i}$ are constant vectors and constant matrices with nonnegative components respectively, $M(t)$ and $K(t)$ are piecewise continuous vector function and matrix function with nonnegative integrable components, respectively. Here the notation $|f(t, x)|=\operatorname{col}\left(\left|f_{1}(t, x)\right|, \ldots,\left|f_{n}(t, x)\right|\right)$ is used and all the inequalities are meant componentwise;

(C) the domain $D_{\beta} \equiv\left\{\xi \in \mathbb{R}^{k} \mid B\left(x_{0}(t, \xi), \beta\right) \subseteq D, t \in[a, b]\right\}$ is non-empty, where $x_{0}(t, \xi)=X(t) P_{G ; k} \xi$,

$$
\begin{aligned}
\beta=\max _{t \in[a, b]}(\mid X(t) & \left(G^{+}+\left(R(t)-G^{+} R_{2}\right) P_{G_{k}^{*}}^{*} R_{1}^{-1} P_{G^{*} ; k}\right) \alpha \mid \\
& \left.+\int_{a}^{b}|L(t, s)| M(s) d s+\sum_{i=1}^{p}\left|L\left(t, \tau_{i}+0\right) m_{i}\right|\right)
\end{aligned}
$$

and $B(y, \varrho)=\left\{x \in \mathbb{R}^{n}:|x-y| \leq \varrho\right\}$ for all $y, \varrho \in \mathbb{R}^{n}$

(D) the maximum eigenvalue of the following matrix $Q$ is less then one:

$$
Q=\sup _{t \in[a, b]}\left\{\int_{a}^{b}|L(t, s)| K(s) d s+\sum_{i=1}^{p}\left|L\left(t, \tau_{i}+0\right)\right| K_{i}\right\} .
$$

We also consider $k$-parametric family of mappings $\mathscr{L}_{\xi}: P C\left([a, b] \backslash\left\{\tau_{i}\right\}_{i=1}^{p}, \mathbb{R}^{n}\right) \rightarrow$ $P C\left([a, b] \backslash\left\{\tau_{i}\right\}_{i=1}^{p}, \mathbb{R}^{n}\right)$ and vector functional $\mu: P C\left([a, b] \backslash\left\{\tau_{i}\right\}_{i=1}^{p}, \mathbb{R}^{n}\right) \rightarrow \mathbb{R}^{k}$ defined by the relations

$$
\begin{aligned}
\left(\mathscr{L}_{\xi} x\right)(t) \stackrel{\text { def }}{=} X(t) P_{G ; k} \xi & +X(t)\left(G^{+}+\left(R(t)-G^{+} R_{2}\right) P_{G_{k}^{*}}^{*} R_{1}^{-1} P_{G^{*} ; k}\right) \alpha \\
& +\int_{a}^{b} L(t, s) f(s, x(s)) d s+\sum_{i=1}^{p} L\left(t, \tau_{i}+0\right) I_{i}\left(x\left(\tau_{i}\right)\right)
\end{aligned}
$$


and

$$
\mu(x) \stackrel{\text { def }}{=} P_{G^{*} ; k}\left(\alpha-\int_{a}^{b} Z(s) f(s, x(s)) d s-\sum_{i=1}^{p} Z\left(\tau_{i}+0\right) I_{i}\left(x\left(\tau_{i}\right)\right)\right) .
$$

Lemma 2. Let the linear homogeneous boundary value problem (2.2), (2.4) has $k, 1 \leq k \leq n$, linearly independent solutions. Then:

(1) If $\varphi \in P C_{\mathrm{loc}}^{1}\left([a, b] \backslash\left\{\tau_{i}\right\}_{i=1}^{p}, \mathbb{R}^{n}\right)$ is a solution of the boundary value problem (2.2), (3.1) then there exists $\xi \in \mathbb{R}^{k}$ such that $\varphi$ is a solution of the system of equations

$$
\begin{aligned}
& x=\mathscr{L}_{\xi} x, \\
& \mu(x)=0 .
\end{aligned}
$$

Moreover, the initial value of the solution is

$$
\varphi(a)=P_{G_{k}} \xi+G^{+}\left(\alpha-\int_{a}^{b} Z(s) f(s, \varphi(s)) d s+\sum_{i=1}^{p} Z\left(\tau_{i}+0\right) I_{i}\left(\varphi\left(\tau_{i}\right)\right)\right) .
$$

(2) If $\varphi \in P C\left([a, b] \backslash\left\{\tau_{i}\right\}_{i=1}^{p}, \mathbb{R}^{n}\right)$ is a solution of the system of equations (3.3), (3.4) with some $\xi \in \mathbb{R}^{k}$ then $\varphi \in P C_{\mathrm{loc}}^{1}\left([a, b] \backslash\left\{\tau_{i}\right\}_{i=1}^{p}, \mathbb{R}^{n}\right)$ and $\varphi$ is a solution of the boundary value problem (2.2), (3.1).

Proof. Necessity. Let $\varphi$ be a solution of the problem (2.2), (3.1). Then

$$
\varphi(t) \equiv X(t) \varphi(a)+\int_{a}^{t} X(t, s) f(s, \varphi(s)) d s+\sum_{a \leq \tau_{i}<t} X\left(t, \tau_{i}+0\right) I_{i}\left(\varphi\left(\tau_{i}\right) .\right.
$$

When substituting (3.6) into the boundary condition (2.2) we can see that $\varphi(a)$ is a solution of algebraic system

$$
G \varphi(a)=\alpha-\int_{a}^{b} Z(s) f(s, \varphi(s)) d s-\sum_{i=1}^{p} Z\left(\tau_{i}+0\right) I_{i}\left(\varphi\left(\tau_{i}\right)\right),
$$

and therefore, there exists $\xi \in \mathbb{R}^{k}$ such that (3.5) holds. Consequently, $\mu(\varphi)=0$. Considering this equality, by direct computation it can be verified that $\varphi$ is also a solution of the equation (3.3).

Sufficiency. Let $\xi$ and $\varphi$ be such that equations (3.3) and (3.4) are satisfied. Therefore, $\varphi \in P C_{\text {loc }}^{1}\left([a, b] \backslash\left\{\tau_{i}\right\}_{i=1}^{p}, \mathbb{R}^{n}\right)$, (3.6) fulfils and $\varphi(a)$ satisfies (3.5). From this follows that $\varphi$ is a solution of problem (2.2), (3.1).

For the investigation of the problem of the existence and approximate constructing of the solutions of problem (2.2), (3.1) we consider the $k$-parametric family of sequences of functions given by the formulas $x_{0}(t, \xi)=X(t) P_{G ; k} \xi$ and

$$
x_{m}(t, \xi)=x_{0}(t, \xi)+X(t)\left(G^{+}+\left(R(t)-G^{+} R_{2}\right) P_{G_{k}^{*}}^{*} R_{1}^{-1} P_{G^{*} ; k}\right) \alpha
$$




$$
+\int_{a}^{b} L(t, s) f\left(s, x_{m-1}(s, \xi) d s+\sum_{i=1}^{p} L\left(t, \tau_{i}+0\right) I_{i}\left(x_{m-1}\left(\tau_{i}, \xi\right)\right.\right.
$$

for $m=1,2, \ldots$ and $\xi \in \mathbb{R}^{k}$. Each of these functions satisfies the boundary condition (2.2).

Theorem 1. Assume that for the boundary value problem (2.2), (3.1) the conditions (A)-(D) hold. Then:

(1) for all $\xi \in D_{\beta} \subset \mathbb{R}^{k}$, the operator $\mathscr{L}_{\xi}$ has a fixed point $x^{*}(\cdot, \xi)$ in the set $P C\left([a, b] \backslash\left\{\tau_{i}\right\}_{i=1}^{p}, \mathbb{R}^{n}\right)$, which coincides with the limit function $x^{*}(t, \xi)=$ $\lim _{m \rightarrow \infty} x_{m}(t, \xi)$ of the sequence (3.8) and the following error estimation holds:

$$
\left|x^{*}(t, \xi)-x_{m}(t, \xi)\right| \leq\left(\mathbb{1}_{n}-Q\right)^{-1} Q^{m} \beta ;
$$

(2) the limit function $x^{*}(t, \xi)$ satisfies the boundary condition (2.2) for arbitrary $\xi \in \mathbb{R}^{k}$ and the initial value is

$$
\begin{aligned}
x^{*}(a, \xi)= & P_{G_{k}} \xi+G^{+}\left(\mathbb{1}_{n}-R_{2} P_{G_{k}^{*}}^{*} R_{1}^{-1} P_{G^{*} ; k}\right) \\
& \times\left(\alpha-\int_{a}^{b} Z(s) f\left(s, x^{*}(s, \xi)\right) d s-\sum_{i=1}^{p} Z\left(\tau_{i}+0\right) I_{i}\left(x^{*}\left(\tau_{i}, \xi\right)\right)\right) ;
\end{aligned}
$$

(3) the limit function $x^{*}(t)=x^{*}\left(t, \xi^{*}\right)$ is a solution of the boundary value problem (2.2), (3.1) if and only if $\xi^{*}$ is a solution of the determining equation $\Delta(\xi)=0$, where

$$
\begin{aligned}
\Delta(\xi) & \stackrel{\text { def }}{=} \mu\left(x^{*}(\cdot, \xi)\right) \\
& \stackrel{\text { def }}{=} P_{G^{*} ; k}\left(\alpha-\int_{a}^{b} Z(s) f\left(s, x^{*}(s, \xi)\right) d s-\sum_{i=1}^{p} Z\left(\tau_{i}+0\right) I_{i}\left(x^{*}\left(\tau_{i}, \xi\right)\right)\right) .
\end{aligned}
$$

Proof. Since

$$
\begin{aligned}
&\left|\left(\mathscr{L}_{\xi} x\right)(t)-x_{0}(t, \xi)\right|\left|X(t)\left(G^{+}+\left(R(t)-G^{+} R_{2}\right) P_{G_{k}^{*}}^{*} R_{1}^{-1} P_{G_{k}^{*}}\right) \alpha\right| \\
&+\int_{a}^{b}|L(t, s) f(s, x(s))| d s+\sum_{i=1}^{p}\left|L\left(t, \tau_{i}+0\right) I_{i}\left(x\left(\tau_{i}\right)\right)\right| \leq \beta,
\end{aligned}
$$

from the condition (C) it follows that $\left(\mathscr{L}_{\xi} x\right)(t) \in D$ for all $\xi \in D_{\beta}, x \in P C([a, b] \backslash$ $\left.\left\{\tau_{i}\right\}_{i=1}^{p}, \mathbb{R}^{n}\right)$.

Let $\mathcal{Q}$ be the operator in $P C\left([a, b] \backslash\left\{\tau_{i}\right\}_{i=1}^{p}, \mathbb{R}^{n}\right)$ acting according to the rule

$$
(\mathcal{Q} x)(t)=\int_{a}^{b}|L(t, s)| K(s) x(s) d s+\sum_{i=1}^{p}\left|L\left(t, \tau_{i}+0\right)\right| K_{i} x\left(\tau_{i}\right) .
$$


Then, from (3.8) and the Lipschitz conditions (3.2) we get

$$
\begin{aligned}
\left|x_{m+1}(t, \xi)-x_{m}(t, \xi)\right| & =\left|\left(\mathscr{L}_{\xi}\left(x_{m}(\cdot, \xi)-x_{m-1}(\cdot, \xi)\right)\right)(t)\right| \\
& \leq\left(\mathcal{Q}\left|x_{m}(\cdot, \xi)-x_{m-1}(\cdot, \xi)\right|\right)(t) \\
& \leq\left(\mathcal{Q}^{2}\left|x_{m-1}(\cdot, \xi)-x_{m-2}(\cdot, \xi)\right|\right)(t) \\
& \leq \cdots \\
& \leq\left(\mathcal{Q}^{m}\left|x_{1}(\cdot, \xi)-x_{0}(\cdot, \xi)\right|\right)(t) \\
& \leq\left(\mathcal{Q}^{m} \beta\right)(t),
\end{aligned}
$$

and thus

$$
\begin{aligned}
\left|x_{m+j}(t, \xi)-x_{m}(t, \xi)\right| & \leq \sum_{i=0}^{j-1}\left|x_{m+i+1}(t, \xi)-x_{m+i}(t, \xi)\right| \\
& \leq \sum_{i=0}^{j-1}\left(Q^{m+i} \beta\right)(t) \\
& \leq \sum_{i=0}^{j-1} Q^{m+i} \beta .
\end{aligned}
$$

It follows from the condition (D) that $x_{m}(t, \xi)$ is a Cauchy sequence. Therefore it uniformly converges to a continuous function $x^{*}(t, \xi)$. Passing to the limit as $j \rightarrow \infty$ in (3.12), we prove the error estimation (3.9). Since all functions satisfy the boundary condition (2.2), we conclude that so does the limit function $x^{*}(t, \xi)$.

Taking the limit as $m \rightarrow \infty$ we get that $x^{*}(t, \xi)$ satisfies the equation

$$
\begin{aligned}
x(t)=X(t) P_{G ; k} \xi & +X(t)\left(G^{+}+\left(R(t)-G^{+} R_{2}\right) P_{G_{k}^{*}}^{*} R_{1}^{-1} P_{G_{k}^{*}}\right) \alpha \\
& +\int_{a}^{b} L(t, s) f(s, x(s) d s)+\sum_{i=1}^{p} L\left(t, \tau_{i}+0\right) I_{i}\left(x\left(\tau_{i}\right)\right) .
\end{aligned}
$$

According to the Lemma 2, it means that the limit function $x^{*}(t, \xi)$ is a solution of the boundary value problem (2.2), (3.1) if and only if the condition $\Delta(\xi)=0$ is fulfilled. Further, putting $t=a$ to (3.13) it is easy to see that (3.10) is the initial value of the solution.

The next statement gives us the satisfactory conditions for the existence of the solution of the boundary value problem (2.2), (3.1). This conditions are based upon the properties of the approximations $x_{m}(t, \xi)$ and not upon those of the limit function $x^{*}(t, \xi)$.

Theorem 2. Let us suppose that conditions (A)-(D) hold and furthermore: 
(1) there exists closed convex subset $D^{\prime} \subset D_{\beta} \subset \mathbb{R}^{k}$ such that, for some fixed $m \in \mathbb{N}$, the approximating equation

$$
\begin{aligned}
& \Delta_{m}(\xi) \stackrel{\text { def }}{=} \mu\left(x_{m}(\cdot, \xi)\right) \\
& \stackrel{\text { def }}{=} P_{G_{k}^{*}}\left(\alpha-\int_{a}^{b} Z(s) f\left(s, x_{m}(s, \xi)\right) d s-\sum_{i=1}^{p} Z\left(\tau_{i}+0\right) I_{i}\left(x_{m}\left(\tau_{i}, \xi\right)\right)\right)=0
\end{aligned}
$$

possesses only one isolated solution $\xi=\xi_{0 m}$ of non-zero index;

(2) on the boundary $\partial D^{\prime}$ of the set $D^{\prime}$, the condition

$$
\inf _{\xi \in \partial D^{\prime}}\left|\Delta_{m}(\xi)\right|>Q_{1}\left(\mathbb{1}_{n}-Q\right)^{-1} Q^{m} \beta
$$

is satisfied, where

$$
Q_{1}=\int_{a}^{b}\left|P_{G_{k^{*}}^{*}} Z(s)\right| K(s) d s+\sum_{i=1}^{p}\left|P_{G_{k^{*}}^{*}} Z\left(\tau_{i}+0\right)\right| K_{i} .
$$

Then there exist a solution $x^{*}(t)=x^{*}\left(t, \xi^{*}\right)$ of the boundary value problem (2.2), (3.1).

Proof. We introduce the continuous for $\xi \in \partial D^{\prime}$ and $\theta \in[a, b]$ vector field family

$$
\Delta(\theta, \xi)=\Delta_{m}(\xi)+\theta\left(\Delta(\xi)-\Delta_{m}(\xi)\right), \quad 0 \leq \theta \leq 1,
$$

connecting the vector fields $\Delta(0, \xi)=\Delta_{m}(\xi)$ and $\Delta(1, \xi)=\Delta(\xi)$. Let us assume that there exist $\theta_{0} \in[0,1]$ such that $\Delta\left(\theta_{0}, \xi\right)=0$. Then

$$
\Delta_{m}(\xi)=-\theta_{0}\left(\Delta(\xi)-\Delta_{m}(\xi)\right)
$$

From (3.9), (3.11), (3.14) and the Lipschitz condition (3.2) we have

$$
\begin{aligned}
\mid \Delta(\xi)- & \Delta_{m}(\xi) \mid \\
\leq & \int_{a}^{b}\left|P_{G^{*} ; k} Z(s)\right|\left|f\left(s, x^{*}(s, \xi)\right)-f\left(s, x_{m}(s, \xi)\right)\right| d s \\
& +\sum_{i=1}^{p}\left|P_{G^{*} ; k} Z\left(\tau_{i}+0\right)\right|\left|I_{i}\left(x^{*}\left(\tau_{i}, \xi\right)\right)-I_{i}\left(x_{m}\left(\tau_{i}, \xi\right)\right)\right| \\
\leq & \int_{a}^{b}\left|P_{G^{*} ; k} Z(s)\right| K(s)\left|x^{*}(s, \xi)-x_{m}(s, \xi)\right| d s \\
& +\sum_{i=1}^{p}\left|P_{G^{*} ; k} Z\left(\tau_{i}+0\right)\right|\left|x^{*}\left(\tau_{i}, \xi\right)-x_{m}\left(\tau_{i}, \xi\right)\right| \\
\leq & Q_{1}\left(\mathbb{1}_{n}-Q\right)^{-1} Q^{m} \beta .
\end{aligned}
$$

But in this case from (3.16) we obtain the inequality

$$
\left|\Delta_{m}(\xi)\right| \leq\left|\Delta(\xi)-\Delta_{m}(\xi)\right| \leq Q_{1}\left(\mathbb{1}_{n}-Q\right)^{-1} Q^{m} \beta,
$$


which contradict the condition (3.15). It means that the field family $\Delta(\theta, \xi)$ does not assume the value zero on $\partial D^{\prime}$, therefore vector fields $\Delta(\xi)$ and $\Delta_{m}(\xi)$ are homotopic. It means that the rotation of vector field $\Delta(\xi)$ on the boundary $\partial D^{\prime}$ is also non-zero and consequently $\Delta(\xi)$ assumes the value zero at least in one point $\xi^{*} \in D^{\prime}$.

\section{NONCRITICAL CASE}

In a noncritical case, i. e., when there is only trivial solution of the linear homogeneous problem (2.6), we obtain $\operatorname{det} G \neq 0, P_{G_{k}}=0$ and $\Delta(\xi) \equiv 0$. This means that is not necessary to solve the determining equation.

Theorem 3. Let us suppose that the linear homogeneous boundary value problem (2.6) has only trivial solution, the condition (B) holds, and

(C1) $B\left(\widetilde{x}_{0}(t), \widetilde{\beta}\right) \subseteq D$ for all $t \in[a, b]$, where

$$
\tilde{x}_{0}(t):=X(t) G^{-1} \alpha, \quad t \in[a, b],
$$

$B(y, \varrho)=\left\{x \in \mathbb{R}^{n}:|x-y| \leq \varrho\right\}$ for all $y, \varrho \in \mathbb{R}^{n}$, the vector $\widetilde{\beta}$ is given by the formula

$$
\tilde{\beta}=\max _{t \in[a, b]}\left(\int_{a}^{b}|\widetilde{L}(t, s)| M(s) d s+\sum_{i=1}^{p}\left|\tilde{L}\left(t, \tau_{i}+0\right)\right| m_{i}\right),
$$

and

$$
\tilde{L}(t, s)= \begin{cases}X(t, s)-X(t) G^{-1} Z(s), & 0 \leq s \leq t \leq b, \\ -X(t) G^{-1} Z(s), & 0 \leq t<s \leq b ;\end{cases}
$$

(D1) the greatest eigenvalue of the following matrix $\widetilde{Q}$ is less then one:

$$
\widetilde{Q}:=\sup _{t \in[a, b]}\left\{\int_{a}^{b}|\widetilde{L}(t, s)| K(s) d s+\sum_{i=1}^{p}\left|\widetilde{L}\left(t, \tau_{i}+0\right)\right| K_{i}\right\} .
$$

Then there exist a unique solution $x^{*}(\cdot)$ of the boundary value problem (2.2), (3.1), which is the limit function of the sequence

$$
\begin{aligned}
\tilde{x}_{m}(t)= & X(t) G^{-1} \alpha+\int_{a}^{b} \tilde{L}(t, s) f\left(s, \tilde{x}_{m-1}(s)\right) d s \\
& +\sum_{i=1}^{p} \tilde{L}\left(t, \tau_{i}+0\right) I\left(\tilde{x}_{m-1}\left(\tau_{i}\right)\right), \quad m=1,2, \ldots
\end{aligned}
$$

Moreover, the initial value of $x^{*}(\cdot)$ satisfies the relation

$$
x^{*}(a)=G^{-1}\left(\alpha-\int_{a}^{b} Z(\tau) f\left(\tau, x^{*}(\tau)\right) d \tau-\sum_{i=1}^{p} Z\left(\tau_{i}+0\right) I_{i}\left(x^{*}\left(\tau_{i}\right)\right)\right) .
$$


Proof. It can be carried out in the same way as that of Theorem 1.

\section{REFERENCES}

[1] A. A. Boichuk and A. M. Samoilenko, Generalized inverse operators and Fredholm boundaryvalue problems. Utrecht: VSP, 2004, translated from the Russian by P. V. Malyshev and D. V. Malyshev.

[2] A. Luchka, Proekcionno-iterativnye metody [Projection-iteration methods]. Kiev: "Naukova Dumka", 1993, in Russian.

[3] L. A. Lyusternik and V. I. Sobolev, Kratkii kurs funktsionalnogo analiza [A short course in functional analysis]. Moscow: "Vyssh. Shkola", 1982.

[4] R. Penrose, "A generalized inverse for matrices," Proc. Cambridge Philos. Soc., vol. 51, pp. 406413, 1955.

[5] A. M. Samoı̌lenko and N. A. Perestyuk, Impulsive differential equations, ser. World Scientific Series on Nonlinear Science. Series A: Monographs and Treatises. River Edge, NJ: World Scientific Publishing Co. Inc., 1995, vol. 14.

[6] A. M. Samorlenko and N. I. Ronto, Chislenno-analiticheskie metody issledovaniya reshenii kraevykh zadach [Numerical-analytic methods for investigating the solutions of boundary value problems]. Kiev: "Naukova Dumka", 1986, with an English summary, Edited and with a preface by Yu. A. Mitropol'skiü.

[7] Š. Schwabik, M. Tvrdý, and O. Vejvoda, Differential and integral equations. Boundary value problems and adjoints. Dordrecht: D. Reidel Publishing Co., 1979.

Author's address

\section{I. Korol}

Uzhhorod National University, Mathematical Department, 46 Pidhirna St., 88000 Uzhhorod, Ukraine

E-mail address: korol_ihor@ukr.net 